\title{
Room-Temperature Synthesis of ZnS Nanoparticles Using Zinc Xanthates as Molecular Precursors
}

\author{
Neli Mintcheva ${ }^{1,2, *(\mathbb{D}, \text { Gospodinka Gicheva }}{ }^{1}$, Marinela Panayotova ${ }^{1}\left[{ }^{\mathbb{B}}\right.$ and \\ Sergei A. Kulinich 2,3,*(D) \\ 1 Department of Chemistry, University of Mining and Geology, Sofia 1700, Bulgaria; \\ e_gospodinka@yahoo.com (G.G.); marichim@mgu.bg (M.P.) \\ 2 Research Institute of Science and Technology, Tokai University, Hiratsuka, Kanagawa 259-1292, Japan \\ 3 Department of Mechanical Engineering, Tokai University, Hiratsuka, Kanagawa 259-1292, Japan \\ * Correspondence: nnmintcheva@mgu.bg (N.M.); skulinich@tokai-u.jp (S.A.K.)
}

Received: 26 November 2019; Accepted: 23 December 2019; Published: 1 January 2020

\begin{abstract}
Molecular precursors are suitable starting compounds for preparation of semiconductor nanoparticles (NPs), which allow for control of atomic ratio, composition, monodispersity, and particle size of nanoscaled metal sulfides/oxides. In the present study, we carried out a one-pot synthesis of ZnS NPs in aqueous triethanolamine medium at room temperature, from molecular precursor zinc xanthate as a source of both $\mathrm{Zn}^{2+}$ and $\mathrm{S}^{2-}$ ions. Furthermore, we compared the products obtained from zinc ethylxanthate $\left(\mathrm{Zn}\left(\mathrm{C}_{2} \mathrm{H}_{5} \mathrm{OCS}_{2}\right)_{2}\right)$ and zinc amylxanthate $\left(\mathrm{Zn}\left(\mathrm{C}_{5} \mathrm{H}_{11} \mathrm{OCS}_{2}\right)_{2}\right)$. The as-prepared ZnS NPs were found to crystallize in cubic phase, which usually forms at low temperatures, with the dimension dependent on the xanthate precursor used. The long carbon-chain xanthate $\mathrm{Zn}\left(\mathrm{C}_{5} \mathrm{H}_{11} \mathrm{OCS}_{2}\right)_{2}$ gave spherically shaped NPs with an average diameter of $19 \mathrm{~nm}$, while the NPs that originated from zinc ethylxanthate had a mean size of $\sim 26 \mathrm{~nm}$. Both nanomaterials had surface sulfur vacancies that extended their absorption spectra toward the visible region and reduced the band gap. This allowed both materials to demonstrate photocatalytic performance under visible-light irradiation. Photodegradation of methylene blue over newly prepared ZnS NPs was tested under visible light, demonstrating efficiency of $50 \%-60 \%$ after $180 \mathrm{~min}$.
\end{abstract}

Keywords: ZnS nanoparticles; zinc xanthate; triethanolamine; photocatalysis

\section{Introduction}

During the last decades, semiconductor nanoparticles (NPs) have been extensively explored because such nanomaterials often demonstrate unique properties that are attractive for applications in various fields, such as optics, electronics, biomedicine, sensing, photocatalysis, and so on. The distinctive properties of semiconductor nanomaterials are well known and result from their chemical and phase composition, NP size and shape, surface chemistry, and defects. Zinc sulfide, as a type II-VI semiconductor, is among the widely investigated materials, and many methods have been proposed for the preparation of its nanostructures with tailored characteristics. For example, the hydrothermal method is commonly used for synthesis of nanosized $\mathrm{ZnS}$ from starting reagents such as sodium sulfide, hydrogen sulfide, thiourea, and sulfur powder [1-6]. The NP size and composition can be controlled by changing the $\mathrm{pH}$ and/or adding a stabilizing agent [7-10]. A number of methods have been applied in order to prepare stable and defect-rich nanomaterials with enhanced properties, such as the microwave irradiation technique [11,12], low-temperature microemulsion [13,14], aerosol microprecipitation [15], capping ZnS NPs with polymer [16], or even biological methods like Stevia-assisted precipitation [17] and sulfate reduction by leaf extract [18]. 
Among the chemical methods for preparation of semiconductor NPs, high-temperature thermolysis of a molecular precursor is a useful approach to achieve monodispersed NPs with well-controlled size [19-24]. Atomic rearrangement takes place throughout the NP formation process from a single-source compound, resulting in a uniform distribution of metal ions and retention of the initial stoichiometry in the final product. In parallel, a stepwise decomposition of the precursor allows for the control of nucleation and crystal growth of NPs. Moreover, ligands strongly coordinated to the metal-ion center of transition metal complexes may affect the NP formation processes and change the crystal phase of the formed NPs, as previously shown by Cheng et al. [19]. Coordinated organic molecules of the precursor are able to act as in-situ capping agents for created NPs [20]. As an example, Liu et al. reported on the use of glutathione as both ligand and sulfur source for luminescent CdS NPs with tunable color [21].

Thermal decomposition of coordination compounds with S-containing ligands (such as dithiocarbamate, dithiocarbonate (xanthate), trithiocarbonate, thiosemicarbazone, and thiobiuret), which serve as sulfide sources, has already been applied to produce metal sulfide NPs with good crystallinity, narrow size distribution, and tunable dimensions [22-25]. For instance, a hot-injection route, which uses thermolysis of metal $(\mathrm{Zn}, \mathrm{Cd}, \mathrm{Hg})$ complexes in alkylamine at $230-280{ }^{\circ} \mathrm{C}$, was shown to lead to formation of monodispersed metal sulfide NPs wrapped with amines [26-30]. Interestingly, aggregates of CdS with sizes of 140-200 nm were obtained from complex Cd(ethylxanthate) 2 , while NPs with diameters of 4-7 nm were formed from diamine-ethylxanthate complex of $\mathrm{Cd}(\mathrm{II})$, indicating the protective role of strongly coordinated diamine ligand for the metal center [31]. Furthermore, cadmium ethylxanthate has been applied to form CdS NPs under different reaction conditions [32-34]. Nair et al. synthesized CdS NPs capped with tri- $n$-octylphosphine oxide (TOPO) through the thermolysis of cadmium ethylxanthate at $280^{\circ} \mathrm{C}$ in inert atmosphere [32]. Multipodal CdS nanostructures were formed from the same single precursor in ethylenediamine solution in a temperature range of $100-180{ }^{\circ} \mathrm{C}$ [33], while long-chain alkyl xanthates of cadmium dissolved in hexadecylamine were found to give CdS NPs upon heating to temperatures $70-120{ }^{\circ} \mathrm{C}$ [34].

It is worth noting that all the above syntheses were carried out at elevated temperatures and typically in inert atmosphere. Pradham et al. reported on the production of metal sulfide crystalline particles with tunable size and shape via metal alkyl xanthates by employing alkylamines as solvents, which were found to play a crucial role in the synthesis, significantly lowering the decomposition temperature of metal xanthate precursors [23]. This finding stimulated us to explore the effect of hydroxyl alkyl amines on the formation of metal sulfide NPs with metal xanthate as a molecular precursor. Therefore, our work aimed to achieve formation of metal sulfide NPs from a xanthate source in ambient conditions and with water as medium. To the best of our knowledge, little information on ZnS NPs obtained from zinc xanthates is available to date $[35,36]$, which is why it is worth exploring how such nanostructures can be controlled under different reaction conditions.

Hence, in this work, we studied the preparation of ZnS NPs from a molecular precursor zinc ethylxanthate $\left(\mathrm{Zn}\left(\mathrm{C}_{2} \mathrm{H}_{5} \mathrm{OCS}_{2}\right)_{2}\right)$ or zinc amylxanthate $\left(\mathrm{Zn}\left(\mathrm{C}_{5} \mathrm{H}_{11} \mathrm{OCS}_{2}\right)_{2}\right)$ as a source of sulfide and zinc ions in reaction with triethanolamine $\left(\mathrm{N}\left(\mathrm{C}_{2} \mathrm{H}_{4} \mathrm{OH}\right)_{3}\right)$, which acted as both nucleophile and a capping agent at room temperature. We report a facile method for the preparation of ZnS NPs with tunable size depending on the length of the carbon chain in the xanthate molecule used as the sulfide source. Considering the remarkable interest in applying $\mathrm{ZnS}$ nanostructures in diverse photocatalytic reactions under visible-light irradiation [37,38], upon characterizing them properly, we then tested the performance of the as-prepared nanomaterials in degradation of methylene blue (MB), which was used as a model compound simulating organic pollutants in wastewaters. 


\section{Materials and Methods}

\subsection{Reagents and Materials}

Potassium ethylxanthate $\left(\mathrm{C}_{2} \mathrm{H}_{5} \mathrm{OCS}_{2} \mathrm{~K}\right.$; Acros Organics, Geel, Belgium), potassium amylxanthate $\left(\mathrm{C}_{5} \mathrm{H}_{11} \mathrm{OCS}_{2} \mathrm{~K}\right.$; TCI, Tokyo, Japan), $\mathrm{Zn}\left(\mathrm{NO}_{3}\right)_{2} \cdot 6 \mathrm{H}_{2} \mathrm{O}$ (Teocom, Sofia, Bulgaria), triethanolamine $\left(\mathrm{N}\left(\mathrm{C}_{2} \mathrm{H}_{4} \mathrm{OH}\right)_{3}\right.$; Teocom, Sofia, Bulgaria), and ethanol (Teocom, Sofia, Bulgaria) were all analytical grade reagents purchased from the corresponding manufacturers and used as supplied and without any further purification.

\subsection{Synthesis of $\mathrm{Zn}\left(\mathrm{C}_{2} \mathrm{H}_{5} \mathrm{OCS}_{2}\right)_{2}$ and $\mathrm{Zn}\left(\mathrm{C}_{5} \mathrm{H}_{11} \mathrm{OCS}_{2}\right)_{2}$}

At room temperature, $0.7437 \mathrm{~g}(2.50 \mathrm{mmol})$ of $\mathrm{Zn}\left(\mathrm{NO}_{3}\right)_{2} \cdot 6 \mathrm{H}_{2} \mathrm{O}$ dissolved in $10 \mathrm{~mL}$ of distilled water was added into $30 \mathrm{~mL}$ of aqueous potassium ethylxanthate with $0.8000 \mathrm{~g}$ (5.00 mmol) of solute. The obtained precipitate of $\mathrm{Zn}\left(\mathrm{C}_{2} \mathrm{H}_{5} \mathrm{OCS}_{2}\right)_{2}$ was filtrated, washed with water and ethanol subsequently, and then dried in air. In a similar way, the powder of $\mathrm{Zn}\left(\mathrm{C}_{5} \mathrm{H}_{11} \mathrm{OCS}_{2}\right)_{2}$ was prepared using potassium amylxanthate $(1.012 \mathrm{~g}(5.0 \mathrm{mmol}))$. Both rinsed and dried compounds were then used as precursors for one-pot synthesis of ZnS NPs.

\subsection{Preparation of ZnS Nanoparticles}

In a typical procedure, $0.2156 \mathrm{~g}(0.70 \mathrm{mmol})$ of $\mathrm{Zn}\left(\mathrm{C}_{2} \mathrm{H}_{5} \mathrm{OCS}_{2}\right)_{2}$ or $0.2745 \mathrm{~g}(0.70 \mathrm{mmol})$ of $\left.\mathrm{Zn}\left(\mathrm{C}_{5} \mathrm{H}_{11} \mathrm{OCS}_{2}\right)_{2}\right)$ was added to $6 \mathrm{~mL}$ of triethanolamine and $6 \mathrm{~mL}$ of distilled water to prepare samples $\mathrm{ZnS}(1)$ and $\mathrm{ZnS}(2)$, respectively. The reaction mixture was stirred at room temperature for three days until a pale yellowish suspension was observed. The obtained NPs were centrifuged at 13,500 rpm for $10 \mathrm{~min}$ and separated from the supernatant. The precipitate was washed three times with distilled water and two times with ethanol to get rid of unreacted amine and other by-products. The products, $\mathrm{ZnS}(1)$ and $\mathrm{ZnS}(2)$, were dried in air and used for further characterization and tests.

\subsection{Characterization of $\mathrm{ZnS}$ Nanoparticles}

The dry powders of samples $\mathrm{ZnS}(1)$ and $\mathrm{ZnS}(2)$ were used for X-ray diffraction (XRD), X-ray photoelectron spectroscopy (XPS), scanning electron microscopy (SEM), Fourier transform infrared (FTIR) spectroscopy, and photocatalytic tests. They were redispersed in ethanol by means of sonication, after which they were drop-cast onto Si wafers for XRD, XPS, and SEM measurements, while more diluted dispersions were used for UV-Vis absorption spectroscopy.

The XRD patterns of the products were recorded on a Bruker instrument (D2 Phaser) with $\mathrm{Cu} / \mathrm{Ni}$ radiation $(\lambda=0.154184 \mathrm{~nm})$ at room temperature. UV-Vis absorption spectra were obtained on a BOECO S-220 spectrophotometer. Field-emission SEM (FE-SEM) images were taken on a Hitachi S-4800 microscope. The XPS measurements were carried out on an ESCAlab-MkII spectrometer (VG Scientific). The FTIR spectra of the samples in KBr tablets were recorded in the range $4000-400 \mathrm{~cm}^{-1} \mathrm{at}^{-}$ a resolution of $1 \mathrm{~cm}^{-1}$ using a Nicolet 6700 FTIR spectrometer.

\subsection{Photocatalytic Tests of ZnS Nanoparticles}

The photocatalytic experiments were performed using $100 \mathrm{~mL}$ of aqueous methylene blue (MB) solution (with concentration $8 \times 10^{-6} \mathrm{~mol} / \mathrm{L}$ ) and $10 \mathrm{mg}$ of each catalyst according to previously described methodology [39]. Prior to irradiation with visible light, the mixture was stirred in the dark for $30 \mathrm{~min}$ to achieve adsorption-desorption equilibrium for each sample tested. The light source was a tungsten halogen lamp (power $1000 \mathrm{~W}$ ) fixed at a distance of $40 \mathrm{~cm}$ above the beaker with the tested mixture. The temperature of the liquid was controlled at $25-26{ }^{\circ} \mathrm{C}$ by means of an ice bath. After each $30 \mathrm{~min}$ interval, $4 \mathrm{~mL}$ of the dispersion was taken, centrifuged, and analyzed by UV-Vis spectroscopy, after which the analyzed aliquot was redispersed by sonication and returned to the beaker for further reaction. 


\section{Results and Discussion}

The reactions between metal xanthates and excess of tertiary amine were carried out for a prolonged time at room temperature. The NPs of samples $\mathrm{ZnS}(1)$ and $\mathrm{ZnS}(2)$ were formed by slow decomposition of zinc ethylxanthate and zinc amylxanthate, respectively, in the presence of triethanolamine, as shown in Scheme 1 below:

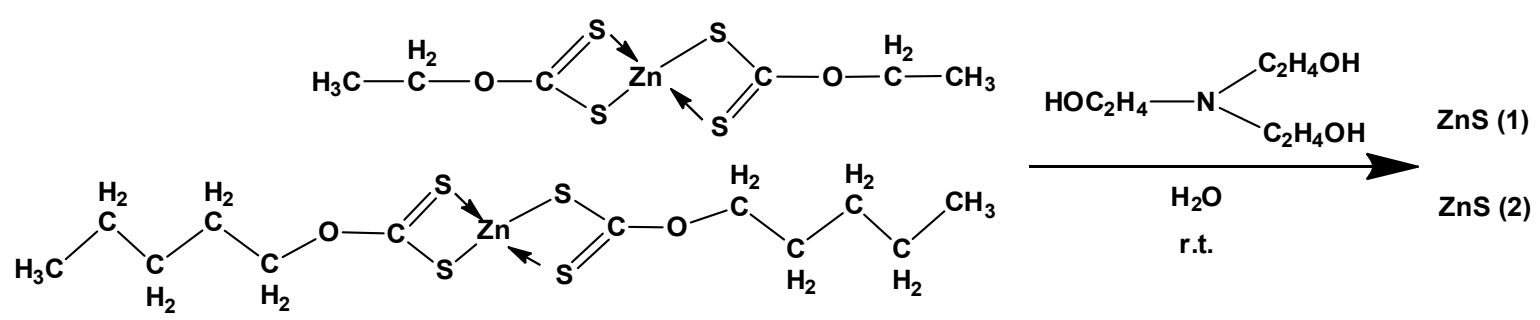

Scheme 1. Reaction conditions for preparation of samples $\mathrm{ZnS}(1)$ and $\mathrm{ZnS}(2)$.

FE-SEM images of sample ZnS(1), prepared from zinc ethylxanthate, and sample ZnS(2), prepared from zinc amylxanthate, are presented in Figure 1 as panels (a) and (b), respectively. It can be seen that both samples had nearly monodispersed spherically shaped particles, with mean sizes of $26 \mathrm{~nm}$ for $\mathrm{ZnS}(1)$ and $19 \mathrm{~nm}$ for $\mathrm{ZnS}(2)$. The size distribution was within the narrow ranges of 15-40 $\mathrm{nm}$ and 10-30 $\mathrm{nm}$ for $\mathrm{ZnS}(1)$ and $\mathrm{ZnS}(2)$, respectively (see histograms in Figure 1c).

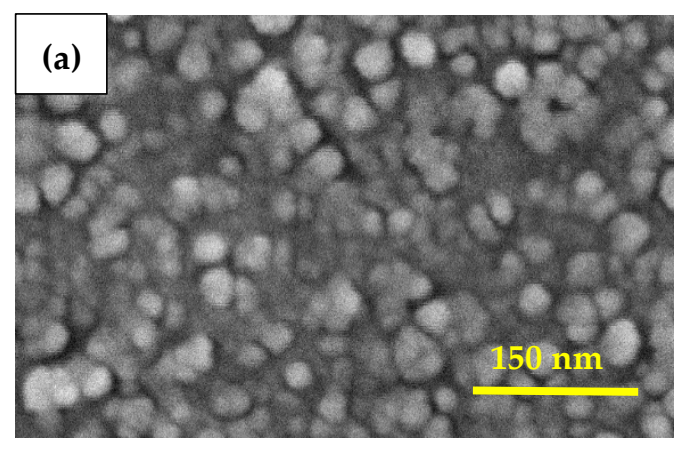

\section{(c)}

ZnS(1)

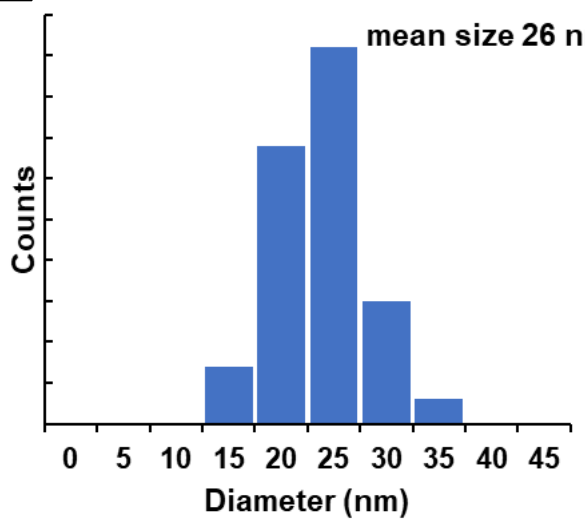

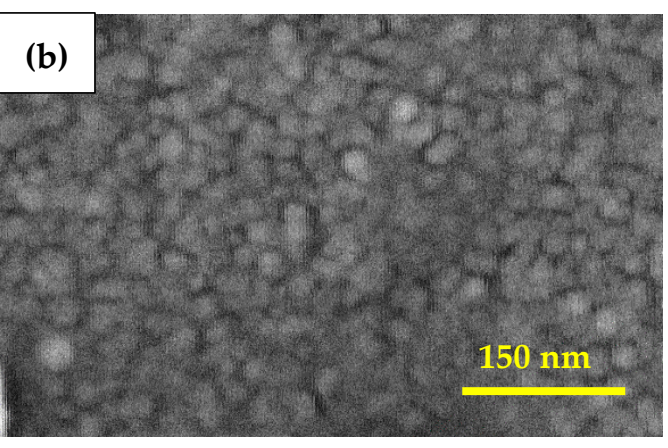

ZnS(2)

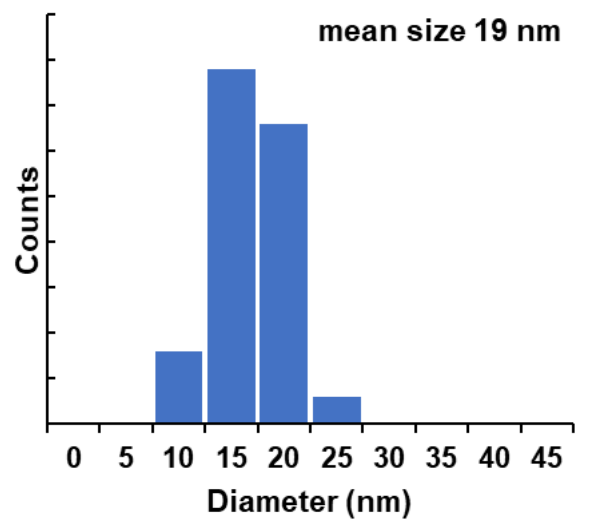

Figure 1. Field-emission scanning electron microscopy (FE-SEM) images of the prepared ZnS NPs: (a) sample ZnS(1) prepared using zinc ethylxanthate; (b) sample ZnS(2) prepared using zinc amylxanthate; (c) Histograms of particle size distribution for samples ZnS(1) and ZnS(2).

The formation of uniform $\mathrm{ZnS}$ nanospheres could be ascribed to triethanolamine molecules, which acted as capping agent and prevented further aggregation of NPs that were forming. Triethanolamine is also believed to slow the release rate of $\mathrm{S}^{2-}$ ions coming from xanthate and contribute to controllable 
growth of ZnS nanocrystals. As a nucleophile, triethanolamine was able to attack the positively charged carbon center in xanthate, thereby causing cleavage of the $C-S$ bond and elimination of the sulfide ion. Then, the sulfide ions generated in situ reacted with zinc ions, forming tiny nanocrystals of ZnS. As clearly seen in Figure 1, the ZnS NPs were smaller when zinc amylxanthate was used as a precursor. We suppose that kinetic factors were responsible for the observed size dependence. In the case of amylxanthate, the nucleophilic reaction was slower for two reasons: (i) the steric hindrance of the five-member-carbon chain in amylxanthate and (ii) the lower partial positive charge on the central carbon atom in amylxanthate owing to a greater inductive effect of its long-chain alkyl group. These two factors resulted in a slower release of sulfide ions from amylxanthate, which in turn limited the size of the growing ZnS NPs.

The XRD patterns of samples ZnS(1) and ZnS(2) are shown in Figure 2, revealing single-phase ZnS materials. The three broad diffraction peaks observed in both patterns correspond to the crystallographic planes (111), (220), and (311) of cubic ZnS and are in agreement with the standard pattern (JCPDS card no. 05-0566). Using the Scherrer equation given below, crystallite sizes were calculated from the XRD patterns:

$$
L=\frac{0.9 \lambda}{B \cos \theta}
$$

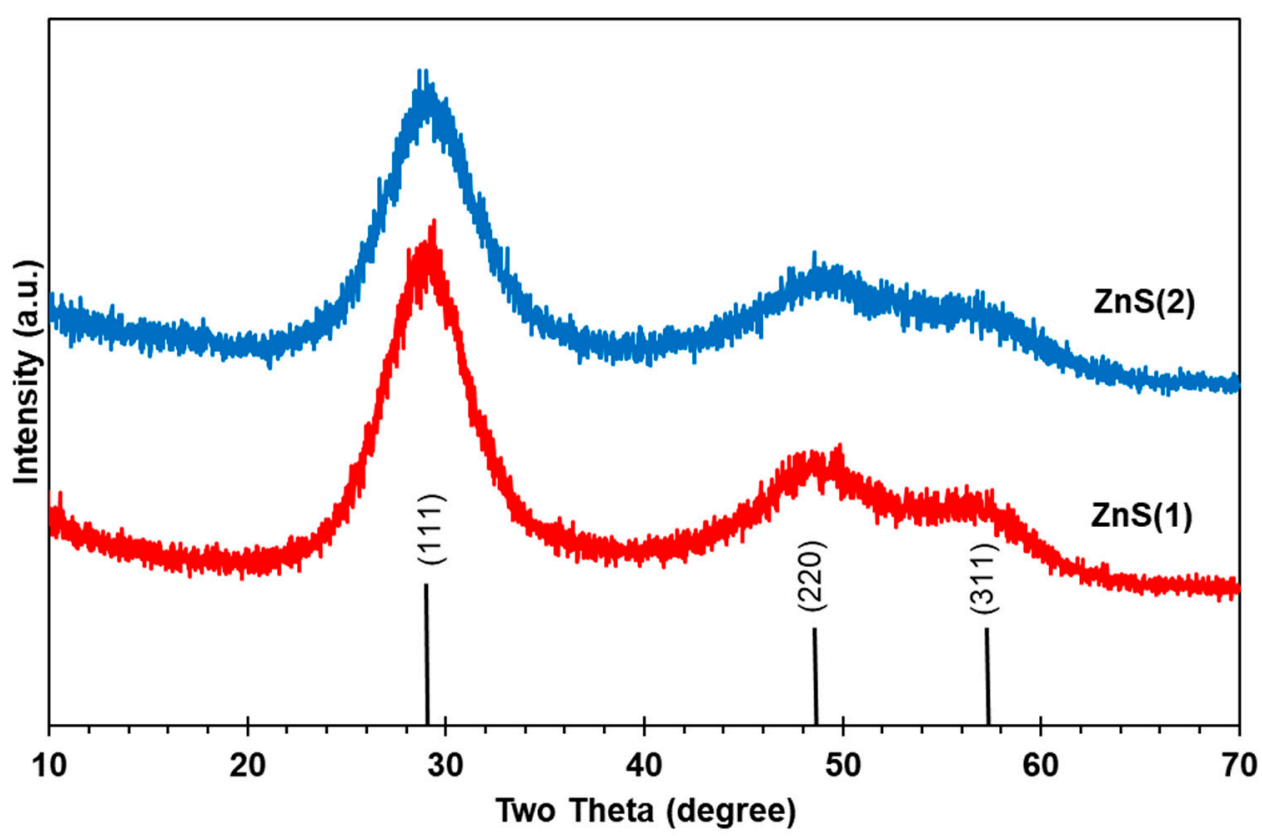

Figure 2. X-ray diffraction (XRD) patterns of samples $\mathrm{ZnS}(1)$ and $\mathrm{ZnS}(2)$.

In this formula, $L$ is the coherence length, $\lambda$ is the wavelength of the $X$-ray radiation used $(0.15406$ $\mathrm{nm}), B$ is the full width at half maximum (FWHM) of the most intense peak, and $\theta$ is the angle of diffraction. The relationship between the coherence length $L$ and the diameter of a spherical NP $(D)$ is known to be $D=4 L / 3[4,9]$. The calculated crystallite diameters for samples $\mathrm{ZnS}(1)$ and $\mathrm{ZnS}(2)$ were 2.8 and $3.2 \mathrm{~nm}$, respectively. Sample $\mathrm{ZnS}(2)$ had a slightly bigger crystallite size and showed a lower degree of crystallinity, as revealed by its broader peaks in the XRD pattern (see blue pattern in Figure 2) [1]. Owing to the very small dimensions and the high surface energy of ZnS crystallites in both samples, they easily aggregated to bigger polycrystalline NPs, as can be seen in the transmission electron microscopy (TEM) image of sample $\mathrm{ZnS}(1)$ in Figure 3. 


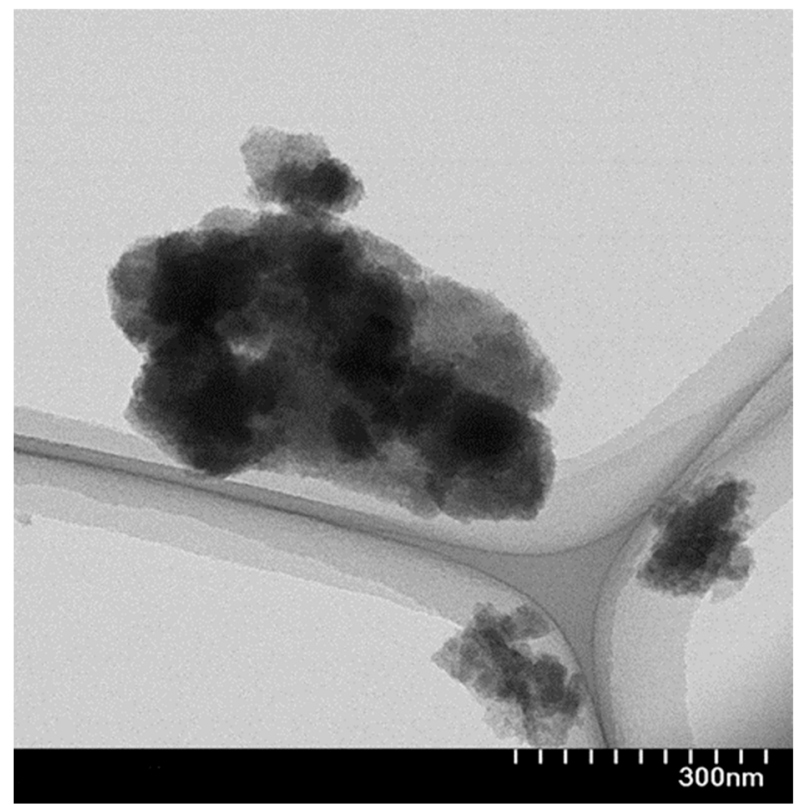

Figure 3. Transmission electron microscopy (TEM) image of sample ZnS(1).

The UV-Vis spectra of samples $\mathrm{ZnS}(1)$ and $\mathrm{ZnS}(2)$ dispersed in ethanol are shown in Figure 4. A broad long-wavelength absorption band was seen for both samples, alongside with distinct exciton absorption peaks observed at 306 and $310 \mathrm{~nm}$ for samples $\mathrm{ZnS}(1)$ and $\mathrm{ZnS}(2)$, respectively. The blue shift of these peaks in comparison with the absorption band at $336 \mathrm{~nm}$ for bulk $\mathrm{ZnS}$ material is attributed to the quantum confinement effect that is well known for nanosized particles $[40,41]$. Similar UV-Vis absorption spectra registered for $3 \mathrm{~nm} \mathrm{ZnS}$ nanocrystals capped with polyvinyl alcohol (PVA), polyvinylpyrrolidone (PVP), and cetyltrimethylammonium bromide (CTAB) were recently presented in [10]. The band gap energy $\left(E_{\mathrm{g}}\right)$ can be calculated using Plank's equation, $E=h c / \lambda$, where $h$ is the Plank constant, $c$ is the speed of light, and $\lambda$ is the wavelength at the absorption maximum $[5,40]$. Using this approach, the $E_{\mathrm{g}}$ values for samples $\mathrm{ZnS}(1)$ and $\mathrm{ZnS}(2)$ were found to be 4.05 and $4.00 \mathrm{eV}$, respectively, while bulk $\mathrm{ZnS}$ is known to have a band gap equal to $3.66 \mathrm{eV}$ [28,42]. Generally, as a result of the quantum confinement effect, the band gap of semiconductor NPs increases with a decrease in NP size, which was indeed observed for both samples.
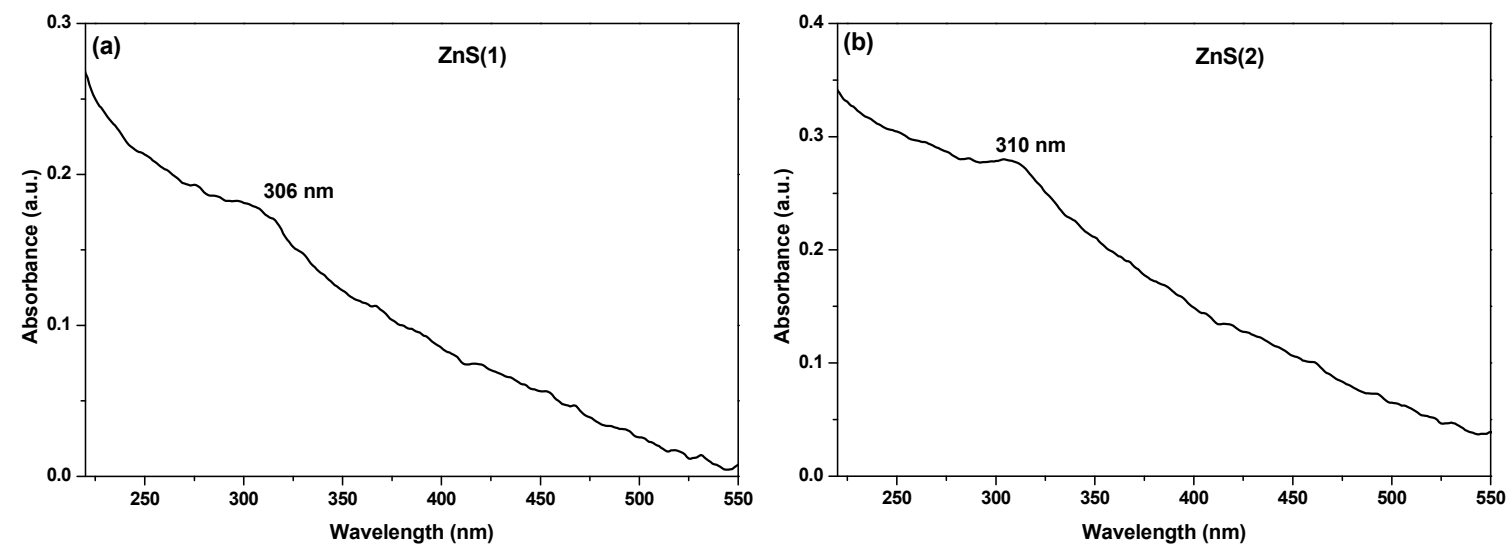

Figure 4. UV-Vis absorption spectra of samples (a) ZnS(1) and (b) ZnS(2).

The elemental composition of the samples and oxidation states of atoms were determined by XPS analysis. Only $\mathrm{Zn}, \mathrm{S}, \mathrm{C}, \mathrm{O}$, and $\mathrm{N}$ atoms were found on the surface of the analyzed NPs, as revealed by XPS survey scans (not shown here). The signals for $\mathrm{C}$ and $\mathrm{O}$ arose not only from environmental contaminations but also from triethanolamine molecules capping the ZnS NPs. Table 1 shows the 
atomic percentage of all elements detected in the samples. As can be seen, both nanomaterials had somewhat larger atomic percentage of $\mathrm{Zn}$ than that of $\mathrm{S}$ atoms. This indicates nonstoichiometric composition and suggests formation of sulfur vacancies in the sample structures. The latter vacancies are expected to be responsible for enhanced photocatalytic activity of the samples, as discussed below.

Table 1. Elemental analysis based on X-ray photoelectron spectroscopy (XPS) data for samples ZnS(1) and $\mathrm{ZnS}(2)$.

\begin{tabular}{cccc}
\hline Sample & Peak & Position & Atomic \% \\
\hline \multirow{3}{*}{ ZnS(1) } & C 1s & 285.2 & 28.18 \\
& N 1s & 399.7 & 3.65 \\
& O 1s & 530.9 & 11.53 \\
& S 2p & 161.4 & 25.66 \\
Zn 2p & 1021.4 & 30.98 \\
\hline \multirow{3}{*}{ ZnS(2) } & C 1s & 285.2 & 34.56 \\
& N 1s & 399.5 & 2.82 \\
& O 1s & 531.2 & 14.62 \\
& S 2p & 161.7 & 20.38 \\
& Zn 2p & 1021.6 & 27.63 \\
\hline
\end{tabular}

Figure 5a,b presents the Zn 2p and S 2p XPS spectra of samples ZnS(1) and ZnS(2), respectively. The peaks at 1021.4 and $1044.4 \mathrm{eV}$ were assigned to $\mathrm{Zn} 2 \mathrm{p}_{3 / 2}$ and $\mathrm{Zn} 2 \mathrm{p}_{1 / 2}$ levels and clearly indicated the presence of $\mathrm{Zn}^{2+}$ ions in the crystal lattice of both samples [43]. Liu and coauthors observed these peaks for ZnS NPs at 1022.1 and $1045.0 \mathrm{eV}$ [25]. Wang et al. demonstrated low-energy shift of Zn 2p peaks with increasing amount of sulfur vacancies [3]. This allowed us to conclude that, for samples $\mathrm{ZnS}(1)$ and $\mathrm{ZnS}(2)$, the position of the $\mathrm{Zn} 2 \mathrm{p}$ peaks was also somehow affected by the presence of $\mathrm{S}$ vacancies. Further evidence for $S$ defects can be gained from the $S 2 p$ XPS spectra shown on Figure $5 b$. As can be seen, the spectra were deconvoluted into four components, assigned to $S 2 p_{3 / 2}$ and $S 2 p_{1 / 2}$ levels from two sulfur species. One set was located at $\sim 160.9$ and $\sim 162.1 \mathrm{eV}$ and was assigned to $S^{2-}$ ions ( $S 2 p_{3 / 2}$ and $S 2 p_{1 / 2}$ levels) surrounded by four $\mathrm{Zn}$ ions in a regular tetrahedral geometry in the crystal lattice (denoted as S1 in Figure $5 b$ ). The second doublet of $S 2 p_{3 / 2}$ and $S 2 p_{1 / 2}$ appeared at $\sim 161.5$ and $\sim 162.8 \mathrm{eV}$ and was attributed to sulfur vacancies, more specifically to sulfide ions located in an irregular manner around the Zn ions in the structure of ZnS (denoted as S2 in Figure 5b). The higher binding energy values observed for $S$ vacancies are in accordance with the trend for defect-rich $Z n S$ nanocrystals previously reported in [3].
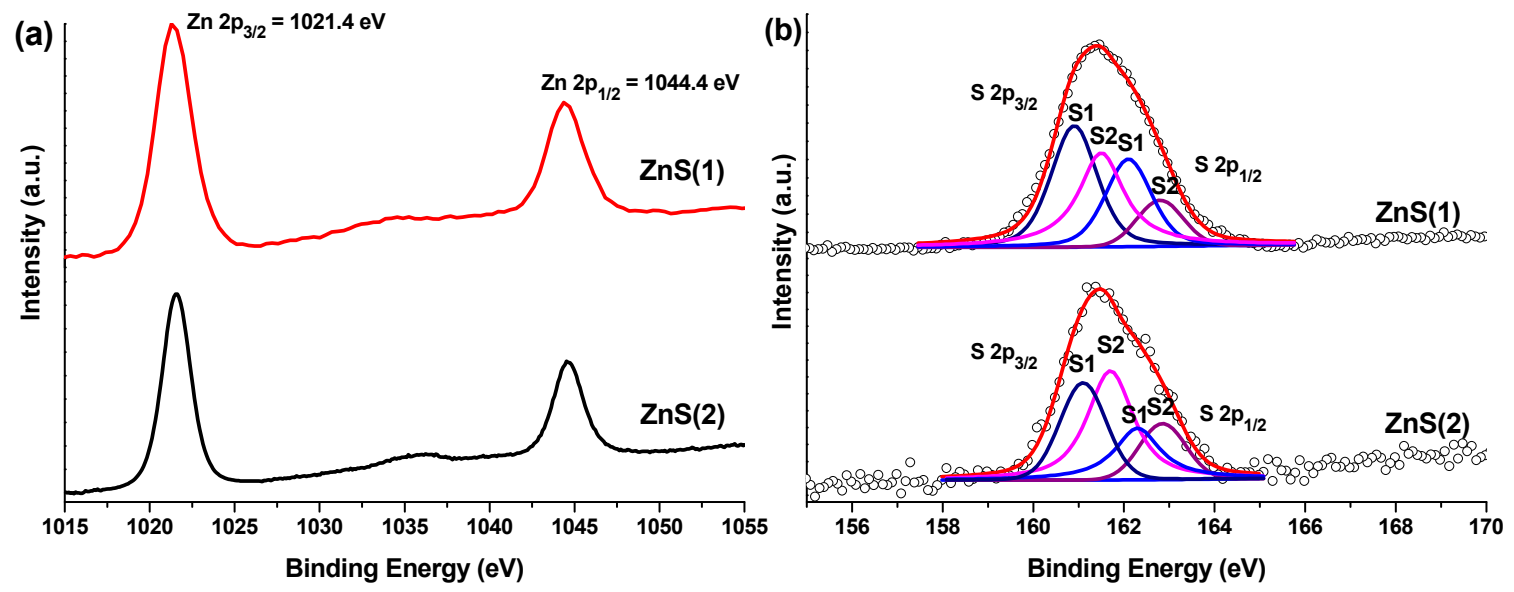

Figure 5. XPS spectra of samples ZnS(1) and ZnS(2): (a) Zn 2p and (b) S 2p.

Additionally, the presence of organic molecules surrounding the ZnS NPs in both samples was confirmed by FT-IR spectroscopy. Table 2 lists the IR bands observed in the spectra of samples ZnS(1) 
and $\mathrm{ZnS}(2)$, along with their assignment, clearly indicating the presence of chemical bonds available in triethanolamine $[44,45]$.

Table 2. Infrared bands and vibrational modes assigned to samples ZnS(1) and ZnS(2).

\begin{tabular}{cccccc}
\hline Peak position $\left(\mathbf{c m}^{-\mathbf{1}}\right)$ & 3430 & 2930 & 1640 & 1400 & $1130-1010$ \\
\hline Assignments & $v_{\mathrm{O}-\mathrm{H}}$ & $v_{\mathrm{C}-\mathrm{H}}$ & $\delta_{\mathrm{O}-\mathrm{H}}$ & $\delta_{\mathrm{C}-\mathrm{H}}$ & $v_{\mathrm{C}-\mathrm{N}}, v_{\mathrm{C}-\mathrm{C},}, v_{\mathrm{C}-\mathrm{O}}$ \\
\hline
\end{tabular}

The photocatalytic activity of both samples was evaluated as they were dispersed in aqueous MB solution and irradiated with visible light. As an organic compound, MB has a stable aromatic structure and is often used as a model substance simulating organic pollutants in water. Its aqueous solution is known to show a strong absorption band at $664 \mathrm{~nm}$ with a shoulder at $610 \mathrm{~nm}$, which are used for monitoring the decomposition of $\mathrm{MB}$ by photocatalytically active materials. The absorption spectra of MB solution in the presence of samples $\mathrm{ZnS}(1)$ and $\mathrm{ZnS}(2)$ were recorded after 0, 30, 60, $90,120,150$, and 180 min under visible-light irradiation. The intensity of the absorption band was found to decrease gradually over time, indicating a steady degradation of the dye. The change in $\mathrm{MB}$ concentration $\left(C / C_{0}\right)$ and the degradation efficiency $\left[\left(C_{0}-C\right) / C_{0} \times 100\right.$, in \%] were determined for both catalysts, which allowed us to plot the percentage of remaining $\mathrm{MB}$ within the irradiation time.

Figure 6 displays how the concentration of MB changed in the presence of both samples $\mathrm{ZnS}(1)$ and $\mathrm{ZnS}(2)$ for as long as $180 \mathrm{~min}$. The degradation efficiency over this period of time was estimated as $51 \%$ and $60 \%$ for samples $\mathrm{ZnS}(1)$ and $\mathrm{ZnS}(2)$, respectively. The nanomaterial produced from xanthate with a longer carbon chain (sample $\mathrm{ZnS}(2)$ ) exhibited somewhat higher photocatalytic activity compared with sample $\mathrm{ZnS}(1)$. This can be explained by its smaller particle size as well as the smaller value of band gap, with the combination of these two factors ensuring advanced photocatalytic performance. Remembering that the newly prepared nanomaterials were both deficient in sulfur, as shown by the XPS chemical analysis (Table 1), one can assume that the formation of sulfur vacancies in the ZnS crystal lattice should also contribute to an increase in visible-light absorption [38]. Previously, Wang et al. used density functional calculations to confirm that sulfur vacancies indeed generated new states located within the band gap (i.e., between the valence and conductive zones) of ZnS, which resulted in a decrease of band gap and enhanced visible-light absorption of $\mathrm{ZnS}$ [3]. Comparison between the areas of peaks S1 and S2 in samples ZnS(1) and ZnS(2), presented in Figure 5b, suggested that the concentration of $\mathrm{S}$ vacancies in sample $\mathrm{ZnS}(2)$ was greater than in sample $\mathrm{ZnS}(1)$. This is believed to be one of the reasons for the higher photocatalytic activity of sample $\mathrm{ZnS}(2)$. The stronger S2 component observed for $\mathrm{ZnS}(2)$ agreed well with the results given in Table 1, where it can be seen that the atomic ratio $\mathrm{Zn}: \mathrm{S}$ was higher for sample $\mathrm{ZnS}(2)$ than for $\mathrm{ZnS}(1)$ (1.36 and 1.21, respectively), indicating that sample $\mathrm{ZnS}(2)$ was more sulfur-deficient.

On the other hand, such vacancies can serve as adsorption sites for oxygen molecules, which are reduced by photogenerated electrons in the conduction band $\left(\mathrm{O}_{2}+\mathrm{e}^{-} \rightarrow \bullet \mathrm{O}_{2}\right)$, as well as adsorption sites for $\mathrm{H}_{2} \mathrm{O}$ molecules, where water undergoes oxidation by holes from the valence band $\left(\mathrm{H}_{2} \mathrm{O}+\mathrm{h}^{+}\right.$ $\rightarrow \mathrm{H}^{+}+\mathrm{OH} \bullet$ ). Thus, the presence of sulfur vacancies should prevent recombination of light-generated electrons and holes, which is a major factor for enhanced photocatalytic activity of the material. In addition, defects in the structure of the prepared nanomaterials may enhance adsorption of $\mathrm{MB}$ molecules on their surface and ensure better contact between MB and the oxidizing species that decomposed this organic dye. 


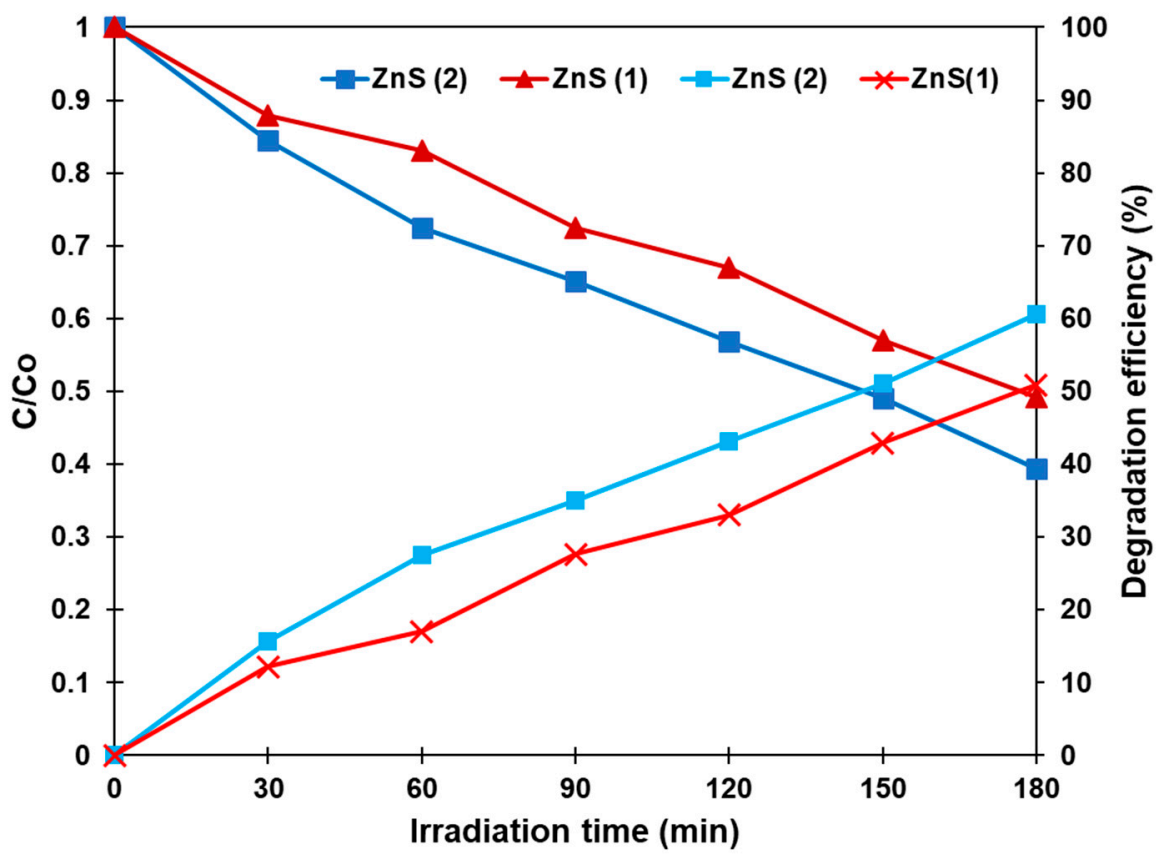

Figure 6. Methylene blue $(\mathrm{MB})$ concentration change $\left(C / \mathrm{C}_{0}\right)$ and degradation efficiency (\%) over samples $\mathrm{ZnS}(1)$ and $\mathrm{ZnS}(2)$ during their irradiation for $180 \mathrm{~min}$. Before illumination, each reaction mixture was kept in the dark for $30 \mathrm{~min}$.

\section{Conclusions}

In this study, for the first time, facile synthesis of ZnS nanoparticles was achieved through the decomposition of zinc xanthates as molecular precursors in the presence of tertiary amine in aqueous solution and at room temperature. Such experimental conditions were shown to favor crystallization of $\mathrm{ZnS}$ in the cubic phase and formation of polycrystalline nanoparticles with sulfur defects on their surface. The particle size was found to be dependent on the carbon-chain length of the organic group in the xanthate: smaller nanoparticles (mean size $19 \mathrm{~nm}$ ) were produced from the xanthate precursor with longer carbon chain, while larger particles $(26 \mathrm{~nm}$ in diameter) were obtained from the xanthate with shorter carbon chain. Both $\mathrm{ZnS}$ samples showed blue shift of their exciton absorption band and higher energy band gap in comparison with bulk $\mathrm{ZnS}$, which is in accordance with the quantum confinement effect. The newly prepared nanomaterials were tested as catalysts for photodegradation of methylene blue in aqueous solution under visible-light irradiation. Higher degradation efficiency was observed for the material with smaller particle sizes. This can be explained by the particle size, smaller band gap energy, and more surface sulfur vacancies, which enhanced the visible-light absorption, reduced the electron-hole recombination, and thus improved the photocatalytic performance of the material.

Author Contributions: Conceptualization, N.M. and G.G.; methodology, G.G.; formal analysis, N.M. and M.P.; investigation, G.G. and M.P.; writing — original draft preparation, N.M.; writing—review \& editing, N.M. and S.A.K. All authors have read and agreed to the published version of the manuscript.

Funding: This research was funded by the National Science Fund of Bulgaria, project number DN-17/20. N.M. would like to thank Tokai University for the exchange academic grant under the Agreement with Bulgarian Ministry of Education and Science.

Acknowledgments: The authors are grateful to Prof. Georgy Tyuliev for XPS measurements and discussion.

Conflicts of Interest: The authors declare no conflict of interest. 


\section{References}

1. Jothi, N.N.S.; Joshi, A.G.; Vijay, R.J.; Muthuvinayagam, A.; Sagayaraj, P. Investigation on one-pot hydrothermal synthesis, structural and optical properties of ZnS quantum dots. Mater. Chem. Phys. 2013, 138, 186-191. [CrossRef]

2. Tezuka, K.; Takagi, H.; Shan, Y.J.; Imoto, H. Simple synthesis of zinc sulfide and cadmium sulfide under hydrothermal conditions. J. Ceram. Soc. Jpn. 2011, 119, 55-59. [CrossRef]

3. Wang, G.; Huang, B.; Li, Z.; Lou, Z.; Wang, Z.; Dai, Y.; Whangbo, M.-H. Synthesis and characterization of $\mathrm{ZnS}$ with controlled amount of $\mathrm{S}$ vacancies for photocatalytic $\mathrm{H}_{2}$ production under visible light. Sci. Rep. 2015, 5, 8544. [CrossRef] [PubMed]

4. Chanu, T.I.; Samanta, D.; Tiwari, A.; Chatterjee, S. Effect of reaction parameters on photoluminescence and photocatalytic activity of zinc sulfide nanosphere synthesized by hydrothermal route. Appl. Surf. Sci. 2017, 391, 548-556. [CrossRef]

5. Ayodhya, D.; Veerabhadram, G. Facile fabrication, characterization and efficient photocatalytic activity of surfactant free ZnS, CdS and CuS nanoparticles. J. Sci. Adv. Mater. Devices 2019, 4, 381-391. [CrossRef]

6. Bhushan, M.; Jha, R.; Bhardwaj, R. Reduced band gap and diffusion controlled spherical n-type ZnS nanoparticles for absorption of UV-Vis region of solar spectrum. J. Phys. Chem. Solids 2019, 135, 109021. [CrossRef]

7. Mansur, A.A.P.; Mansur, H.S.; Ramanery, F.P.; Oliveira, L.C.; Souz, P.P. “Green” colloidal ZnS quantum dots/chitosan nano-photocatalysts for advanced oxidation processes: Study of the photodegradation of organic dye pollutants. Appl. Catal. B 2014, 158, 269-279. [CrossRef]

8. Kole, A.K.; Kumbhakar, P. Cubic-to-hexagonal phase transition and optical properties of chemically synthesized ZnS nanocrystals. Results Phys. 2012, 2, 150-155. [CrossRef]

9. Huo, F.; Wang, Y.; You, C.; Deng, W.; Yang, F.; Pu, Y. Phase- and size-controllable synthesis with efficient photocatalytic activity of ZnS nanoparticles. J. Mater. Sci. 2017, 52, 5626-5633. [CrossRef]

10. Deka, D.C.; Kalita, A.; Bardaloi, S.; Kalita, M.P.C. Influence of capping agent on structural, optical and photocatalytic properties of ZnS nanocrystals. J. Lumin. 2019, 210, 269-275. [CrossRef]

11. Soltani, N.; Saion, E.; Hussein, M.Z.; Erfani, M.; Abedini, A.; Bahmanrokh, G.; Navasery, M.; Vaziri, P. Visible Light-Induced Degradation of Methylene Blue in the Presence of Photocatalytic ZnS and CdS Nanoparticles. Int. J. Mol. Sci. 2012, 13, 12242-12258. [CrossRef] [PubMed]

12. Al-Rasoul, K.T.; Ibrahim, I.M.; Ali, I.M.; Al-Haddad, R.M. Synthesis, structure and characterization of ZnS QDs and using it in photocatalytic reaction. Int. J. Sci. Technol. Res. 2014, 3, 213-217.

13. Liveri, V.T.; Rossi, M.; D'Arrigo, G.; Manno, D.; Micocci, G. Synthesis and characterization of ZnS nanoparticles in water/AOT/n-heptanemicroemulsions. Appl. Phys. A 1999, 69, 369-373. [CrossRef]

14. Calandra, P.; Goffredi, M.; Turco Liveri, V. Study of the growth of ZnS nanoparticles in water: AOT:n-heptane microemulsions by UV-absorption spectroscopy. Colloid. Surf. A 1999, 160, 9-13. [CrossRef]

15. Bednář, J.; Svoboda, L.; Mančík, P.; Dvorský, R. Synthesis of ZnS nanoparticles of required size by precipitation in aerosol microdroplets. Mater. Sci. Technol. 2019, 35, 775-781. [CrossRef]

16. Tiwari, A.; Dhoble, S.J. Stabilization of ZnS nanoparticles by polymeric matrices: Syntheses, optical properties and recent applications. RSC Adv. 2016, 6, 64400-64420. [CrossRef]

17. Alijani, H.Q.; Pourseyedi, S.; Mahani, M.T.; Khatami, M. Green synthesis of zinc sulfide (ZnS) nanoparticles using Stevia rebaudiana Bertoni and evaluation of its cytotoxic properties. J. Mol. Struct. 2019, 1175, 214-218. [CrossRef]

18. Chen, J.; Hub, B.; Zhi, J. Optical and photocatalytic properties of Corymbia citriodora leaf extract synthesized ZnS nanoparticles. Phys. E 2016, 79, 103-106. [CrossRef]

19. Cheng, Y.; Lin, Z.; Lü, H.; Zhang, L.; Yang, B. ZnS nanoparticles well dispersed in ethylene glycol: Coordination control synthesis and application as nanocomposite optical coatings. Nanotechnology 2014, 25, 115601. [CrossRef]

20. Li, Y.; Li, X.; Yang, C.; Li, Y. Ligand-controlling synthesis and ordered assembly of ZnS nanorods and nanodots. J. Phys. Chem. B 2004, 108, 16002-16011. [CrossRef]

21. Liu, Y.; Yin, S.-N.; Yang, S. Glutathione as both ligand and sulfur source for the synthesis of full-color luminescent water-soluble CdS nanocrystals. Mater. Lett. 2017, 196, 260-263. [CrossRef] 
22. Andrew, F.P.; Ajibade, P.A. Metal complexes of alkyl-aryl dithiocarbamates: Structural studies, anticancer potentials and applications as precursors for semiconductor nanocrystals. J. Mol. Struct. 2018, 1155, 843-855. [CrossRef]

23. Pradhan, N.; Katz, B.; Efrima, S. Synthesis of High-Quality Metal Sulfide Nanoparticles from Alkyl Xanthate Single Precursors in Alkylamine Solvents. J. Phys. Chem. B 2003, 107, 13843-13854. [CrossRef]

24. Ramasamy, K.; Malik, M.A.; O’Brien, P.; Raftery, J.; Helliwell, M. Nickel Sulfide Thin Films from Thio- and Dithiobiuret Precursors. Chem. Mater. 2010, 22, 6328-6340. [CrossRef]

25. Liu, W. Low temperature synthesis of hexagonal phase ZnS nanocrystals by thermolysis of an air-stable single-source molecular precursor in air. Mat. Lett. 2006, 60, 551-554. [CrossRef]

26. Onwudiwe, D.C.; Ajibade, P.A. Zn(II), Cd(II) and Hg(II) complexes of N-methyl-N-phenyl dithiocarbamate as single-source precursors for the synthesis of metal sulfide nanoparticles. Mat. Lett. 2011, 65, 3258-3261. [CrossRef]

27. Onwudiwe, D.C.; Strydom, C.; Oluwafemi, O.S.; Songca, S.P. Effect of temperature on the optical and structural properties of hexadecylamine capped ZnS nanoparticles using Zinc(II) N-ethyl-N-phenyldithiocarbamate as single source precursor. Mat. Res. Bull. 2012, 47, 4445-4451. [CrossRef]

28. Pawar, A.S.; Mlowe, S.; Garje, S.S.; Akerman, M.P.; Revaprasadu, N. Zinc thiosemicarbazone complexes: Single source precursors for alkylamine capped ZnS nanoparticles. Inorg. Chim. Acta 2017, 463, 7-13. [CrossRef]

29. Pawar, A.S.; Masikane, S.C.; Mlowe, S.; Garje, S.S.; Revaprasadu, N. Preparation of CdS Nanoparticles from Thiosemicarbazone Complexes: Morphological Influence of Chlorido and Iodido Ligands. Eur. J. Inorg. Chem. 2016, 2016, 366-372. [CrossRef]

30. Onwudiwe, D.C.; Mohammed, A.D.; Strydom, C.A.; Young, D.A.; Jordaan, A. Colloidal synthesis of monodispersed $\mathrm{ZnS}$ and $\mathrm{CdS}$ nanocrystals from novel zinc and cadmium complexes. Superlattice Microst. 2014, 70, 98-108. [CrossRef]

31. Cusack, J.; Drew, M.G.B.; Spalding, T.R. Syntheses and spectroscopy of diamine complexes of Zn(II) and $\mathrm{Cd}(\mathrm{II})$ ethylxanthates and the molecular structures of [M( $\left.\left.\mathrm{S}_{2} \mathrm{COEt}\right)_{2} \mathrm{TMEDA}\right]$ : Formation of CdS nanoparticles from $\left[\mathrm{Cd}\left(\mathrm{S}_{2} \mathrm{COEt}\right)_{2}\right]$ and [Cd $\left.\left(\mathrm{S}_{2} \mathrm{COEt}\right)_{2} \mathrm{TMEDA}\right]$. Polyhedron 2004, 23, 2315-2321. [CrossRef]

32. Nair, P.S.; Radhakrishnan, T.; Revaprasadu, N.; Kolawole, G.; O’Brien, P. Cadmium ethylxanthate: A novel single-source precursor for the preparation of CdS nanoparticles. J. Mater. Chem. 2002, 12, 2722-2725. [CrossRef]

33. Han, Q.; Zhao, J.; Wu, L.; Zhu, J.; Wang, X. Synthesis of CdS multipods from cadmium xanthate in ethylenediamine solution. Particuology 2015, 19, 45-52. [CrossRef]

34. Pradhan, N.; Efrima, S. Single-precursor, one-pot versatile synthesis under near ambient conditions of tunable, single and dual band fluorescing metal sulfide nanoparticles. J. Am. Chem. Soc. 2003, 125, 2050-2051. [CrossRef]

35. Onwudiwe, D.C.; Krüger, T.P.J.; Strydom, C.A. Laser assisted solid state reaction for the synthesis of ZnS and CdS nanoparticles from metal xanthate. Mater. Lett. 2014, 116, 154-159. [CrossRef]

36. Barreca, D.; Tondello, E.; Lydon, D.; Spalding, T.R.; Fabrizio, M. Single-source chemical vapor deposition of zinc sulfide-based thin films from zinc bis(O-ethylxanthate). Chem. Vap. Depos. 2003, 9, 93-98. [CrossRef]

37. Mintcheva, N.; Gicheva, G.; Panayotova, M.; Wunderlich, W.; Kuchmizhak, A.A.; Kulinich, S.A. Preparation and photocatalytic properties of CdS and ZnS nanomaterials derived from metal xanthate. Materials 2019, 12, 3313. [CrossRef]

38. Lee, G.-J.; Wu, J.J. Recent developments in ZnS photocatalysts from synthesis to photocatalytic applications-A review. Powder Technol. 2017, 318, 8-22. [CrossRef]

39. Mintcheva, N.; Aljulaih, A.A.; Wunderlich, W.; Kulinich, S.A.; Iwamori, S. Laser-ablated ZnO nanoparticles and their photocatalytic activity towards organic pollutants. Materials 2018, 11, 1127. [CrossRef]

40. Kumbhojkar, N.; Nikesh, V.V.; Kshirsagar, A.; Mahamuni, S. Photophysical properties of ZnS nanoclusters. J. Appl. Phys. 2000, 88, 6260-6264. [CrossRef]

41. Rossetti, R.; Hull, R.; Gibson, J.M.; Brus, L.E. Excited electronic states and optical spectra of ZnS and CdS crystallites in the $\approx 15$ to $50 \AA$ size range: Evolution from molecular to bulk semiconducting properties. J. Chem. Phys. 1985, 82, 552-559. [CrossRef]

42. Wageh, S.; Ling, Z.S.; Xu-Rong, X. Growth and optical properties of colloidal ZnS nanoparticles. J. Cryst. Growth 2003, 255, 332-337. [CrossRef] 
43. Perkin-Elmer Corporation. Handbook of X-ray Photoelectron Spectroscopy; Physical electronics: Chanhassen, MN, USA, 1992.

44. Coates, J. Interpretation of Infrared Spectra, A Practical Approach. Encyclopedia of Analytical Chemistry; Meyers, R.A., Ed.; John Wiley \& Sons Ltd.: Hoboken, NJ, USA, 2000.

45. Infrared spectrum of triethanolamine. Available online: https://webbook.nist.gov/cgi/cbook.cgi?ID= C102716\&Type=IR-SPEC\&Index=0\#IR-SPEC (accessed on 12 December 2019).

(c) (1)

(C) 2020 by the authors. Licensee MDPI, Basel, Switzerland. This article is an open access article distributed under the terms and conditions of the Creative Commons Attribution (CC BY) license (http://creativecommons.org/licenses/by/4.0/). 